\title{
MATERNAL DIET DURING PREGNANCY AND INTESTINAL MARKERS ARE ASSOCIATED WITH EARLY GUT MICROBIOTA.
}

Selma-Royo, M.; García-Mantrana, I.; Calatayud, M.; Parra-Llorca, A.; Martínez-Costa, C.; Collado, M.C. Maternal diet and intestinal homeostasis influence early gut microbiota. Eur. J. Nutr. 2020.

Accepted July 2020

Impact factor: 4.449

\begin{abstract}
ABTRACT
Diet has an important role in host-microbiome interplay, which may result in intestinal permeability changes and physio pathological effects at a systemic level. Despite the importance of maternal microbiota as the main contributor of the initial microbial seeding, little is known about the effects of maternal diet during pregnancy on maternalneonatal microbiota.

To ascertain the possible associations between maternal dietary intake during pregnancy and neonatal microbiota at birth, and to evaluate the relation with maternal intestinal markers.

In a nested cross-sectional study in the longitudinal MAMI cohort, maternal-neonatal microbiota profiling at birth $(\mathrm{n}=73)$ was assessed by $16 \mathrm{~S}$ rRNA gene sequencing. Maternal intestinal markers as zonulin, intestinal alkaline phosphatase (IAP) activity, and faecal calprotectin were measured in faeces. Furthermore, maternal-neonatal clinical and anthropometric data as well as maternal nutrient intake during pregnancy obtained by FFQ questionnaires were collected.

Maternal diet is associated with both maternal and neonatal microbiota at time of birth, in a delivery mode dependent manner. The existing link between maternal diet, intestinal makers, and neonatal gut microbiota would be mainly influenced by intake of saturated (SFA) and monounsaturated fatty acids (MUFA). Firmicutes members in the neonatal microbiota were positively associated with maternal fat intake, especially SFA and MUFA, and negatively correlated to fibre, proteins from vegetable sources, and vitamins.

Maternal diet during pregnancy, mainly fat intake (SFA and MUFA), was related to intestinal biochemical markers, thus likely shifting microbial transmission to the neonate, and priming the neonatal microbial profile with potential health outcomes.
\end{abstract}

Keywords: maternal diet, intestinal permeability, microbiota. 


\section{INTRODUCTION}

Maternal microbiota represents the most important microbial source for the neonatal microbiota colonization process. Increasing evidence suggests that maternal diet during pregnancy shapes the offspring's microbiota composition and intestinal development in the short and long term [194]. Studies of animal models have highlighted the role of the maternal intake of specific nutrients, such as fibre, which drive neonatal microbiota and suppress intestinal permeability [536].

During pregnancy, the female body adapts its metabolism, hormonal cascades, and immunological events to optimize the growth of the foetus and prepare the body for lactation [132], leading to similar markers to those observed in metabolic syndrome $[132,537]$. Gut microbiota is also modified by these changes, which have several consequences including increasing the energy extraction efficiency from the diet [132]. An increased intestinal permeability has also been reported in pregnant women [538], which may relate to low-grade systemic inflammation in mothers and neonates [539]. Higher gut permeability was observed in overweight pregnant women [540] and those with gestational diabetes mellitus (GDM) risk [541,542] compared to healthy pregnant women.

Most studies of microbiota and dietary patterns have evaluated extreme diets (e.g., Western diet vs. non-Western diet) or diseased individuals (e.g., obese) [543,544]. However, the impact of diet and intestinal permeability during pregnancy on female microbiota and the possible subsequent health consequences are still not fully understood, specifically in healthy cohorts (non-obese) following a healthy standard diet. Few publications have shown that maternal diet can modulate the gut microbiome of the mother or infant [198]. Thus, more information is required to address how nutrient patterns influence maternal and neonate gut microbiota.

This study assesses the association of maternal nutrients intake during gestation on maternal intestinal functional markers and determines its relation to maternalneonatal gut microbiota. The aim is to shed light on the potential factors related to the vertical mother-infant transmission affecting the critical initial contact of the neonate with maternal microbiota and to identify possible "window" for dietary intervention promoting health-associated taxa in the gut of the mother and their offspring. 


\section{MATERIALS AND METHODS}

\section{Study design and volunteers}

A total number of 116 mother-infant dyads from the observational study MAMI birth cohort recruited during 2015-2017 were included in the study. MAMI cohort consist in a prospective mother-infant cohort in the Spanish-Mediterranean area as detailed previously [420]. Samples at birth time were selected to assess the maternal diet association with the neonatal and maternal microbiota.

\section{Clinical, anthropometric, and obstetric characteristics}

Nutritional, anthropometrical and clinical parameters of mother-infant pairs were recorded at birth time. Available data included antibiotic consumption during pregnancy, pre-gestational body mass index (BMI) and weight gain during pregnancy, gestational age, as well as mode of birth, intrapartum antibiotic exposure, neonatal weight and length at birth.

None of the participating volunteers have been diagnosed of any disease; neither were under drug treatment or prebiotics administration, except for antibiotics use during pregnancy and/or at delivery.

\section{Assessment of maternal dietary intake}

Women were asked to fill out in a 140-item validated food questionnaire (FFQ) about their diet during the pregnancy [421] within first week after delivery. FFQ information was analysed using the Nutrients Food Composition Tables developed by the Centro de Enseñanza Superior de Nutrición Humana y Dietética (CESNID), Spain [494]. FFQ has been validated previously [422]. For further analysis, the caloric contribution (in percentage) of each macronutrients to total energy intake, including total protein, those from both animal and vegetable sources, lipids, SFA, mono- and poly-unsaturated fatty acids (MUFA and PUFA), total carbohydrates, polysaccharides and fibre, was calculated. Proteins and carbohydrates, including polysaccharides, was considered to contribute in $4 \mathrm{kcal} / \mathrm{g}$, lipids $9 \mathrm{kcal} / \mathrm{g}$ and fibre, as special carbohydrate, was estimated to have $2 \mathrm{kcal} / \mathrm{gr}$ contribution. 


\section{Maternal-neonatal biological samples}

Maternal-neonatal rectal swabs were collected by trained personnel at delivery room at the hospital [420]. Maternal gut samples were obtained by swabbing a sterile, cotton-tipped swab in the rectum before giving birth in the delivery room. Neonatal sample collection was also obtained by introducing a sterile, cotton-tipped swab in the neonatal rectum after birth on the resus-A-cradle in the delivery room. After collection, samples were immediately stored at $-20^{\circ} \mathrm{C}$ and transported in dry ice within the next 24 hours to the laboratory for storage at $-80^{\circ} \mathrm{C}$ until further analysis.

Faecal samples were homogenized in PBS 1X, vortexed $1 \mathrm{~min}$ and centrifuged at $13,000 \mathrm{~g}$ for $10 \mathrm{~min}$. Supernatants were collected for the intestinal markers quantification while the resulted pellet was used for the total DNA extraction procedure.

\section{Maternal intestinal markers}

Faecal supernatants were used to determine the following intestinal markers: zonulin, Intestinal alkaline phosphatase (IAP) and calprotectin.

Zonulin measurement was performed by commercial ELISA test (MyBioSource, San Diego, CA) according to manufacturer protocol and using a 1:2 dilution sample. Final concentrations were calculated based on each standard curve values, including in duplicate in each batch. IAP activity was quantified by the Alkaline Phosphatase Diethanolamine Activity Kit following the manufacturer's instructions (Sigma-Aldrich, USA) and faecal calprotectin was determined by ELISA assay (Wuhan Fine Biotech, Wuhan, China) according to manufacturer's instruction with a 1:5 dilution of maternal faecal supernatants in sample dilution buffer from the kit reagents.

\section{DNA Extraction and 16S rRNA amplicon sequencing}

Total DNA was isolated from the maternal-neonatal faecal swabs using the Master-Pure DNA Extraction Kit (Epicentre, Madison, WI, United States) by the manufacture's instruction with some modifications. Cell lysis was performed by mechanical disruption using $3-\mu \mathrm{m}$ diameter glass beads $1 \mathrm{~min}$ at $6 \mathrm{~m} / \mathrm{s}$ and a FastPrep 24-5G Homogenizer (MP Biomedicals), followed by a chemical treatment with lysis buffer from the extraction kit, supplemented with lysozyme $(20 \mathrm{mg} / \mathrm{ml})$ and mutanolysine $(5 \mathrm{U} / \mathrm{ml})\left(60 \mathrm{~min}\right.$ at $\left.37^{\circ} \mathrm{C}\right)$. DNA Purification Kit (Macherey-Nagel, Duren, Germany) was used to the DNA purification. The resulting DNA was quantified 
and normalized to 5ng/ul by Qubit 2.0 Fluorometer (Life Technology, Carlsbad, CA, United States).

The microbial composition was determined by sequencing of the V3-V4 variable region of the 16S rRNA gene. The region was amplified using the Illumina adapter overhang nucleotide sequences following Illumina protocol. Nextera XT Index Kit (Illumina, CA, USA) was used for the multiplexing step and a Bioanalyzer DNA 1000 chip (Agilent Technologies, CA, USA) for checking the PCR product quality. Libraries were sequenced using a 2x300 pb paired-end run (MiSeq Reagent kit v3) on a MiSeqIllumina platform (FISABIO sequencing service, Valencia, Spain).

Quality filtering, sequence joining, and chimera removal were achieved using DADA2 pipeline [545]. Taxonomic assignment was performed using Silva v132 database [440] with the addition of the specie level classification. Samples with low relative abundance $(<0.01 \%)$ and those present in less than 5 times in at least $20 \%$ of the samples were filtered. Similarly, sequences from contaminants and those classified as Cyanobacteria and Chloroplast, considered ingested plant material were also removed. Samples with less than 1000 reads $(n=5)$ were also removed from the final data analysis. Bacterial counts were normalized by Total Sum Scaling (TSS).

\section{Statistical analysis}

Microbial sequences were analysed by use of Calypso online platform (v. 8.72) [443], SPSS software [444] and Rstudio environment [445] were used. RStudio was used to perform the PERMANOVA (Adonis) multivariate analysis based on BrayCurtis distance in maternal and neonatal microbiota by the vegan $\mathrm{R}$ package [505]. This $\mathrm{R}$ package was also used for microbial alpha diversity by Shannon (diversity) and Chao1 indexes (richness). The heatmaps between bacterial taxonomic groups and dietary components were performed through "ggplot" package [446]. Indeed, spearman rank correlations between maternal diet and microbiota composition were adjusted by delivery mode. Multivariate analysis of maternal-neonatal microbiota, including Discriminant analysis of Principal Components (DAPC) and Adonis test (PERMANOVA) were also achieved based on Bray-Curtis distance in Calypso online platform.

SPSS software v.25 was used for the spearman correlations and for T-test and Mann-Whitney analysis according to data normality assessed by Shapiro-Wilk test. Chi- 
Squared test $(2 \times 2)$ was performed to assess the significance of the differences in the population characteristics and nutrients intakes in categorical variables.

In order to group the mothers according to their diet, we performed a classification of the mothers for each nutrient according whether their consumption was above or below the average of the study population. The differences between the groups were visualized by discriminant of principal components analysis (DAPC) at the OTU level, and statistical significance was assessed using the Adonis test. DEseq2 was conducted in Calypso platform to found significant differences in maternal and neonatal microbiota according to these classifications. All comparisons performed with DEseq2 was adjusted by FDR method.

\section{Ethical aspects}

All participants received oral and written information about the study and written consent was obtained. The study was approved by the Hospital ethics committees (HECs) (Hospital Universitario y Politécnico La Fe and Hospital Clínico Universitario de Valencia). The study is registered on the ClinicalTrial.gov platform, with the registration number NCT03552939.

\section{RESULTS}

\section{Characteristics of study participants}

A total number of 73 mothers-neonates were finally included according to the full availability of biological samples, clinical and dietary data for the cross-sectional study (Supplementary file 1). The maternal population showed a median of 35 years old, a pre-gestational BMI of $21.8 \mathrm{~kg} / \mathrm{m}^{2}$, and a weight gain during pregnancy of around $12.68 \pm 4.28 \mathrm{~kg}$, which is in the range recommended by the Institute of Medicine [438] (Table 1).

C-section births represented 38.4\% (28/73), and no significant differences in pre-pregnancy BMI and weight gain over pregnancy were found between modes of birth (vaginal vs. C-section). Significant differences in maternal antibiotic treatment at delivery $(p<0.001)$ and gestational age $(p=0.018)$ were found between the vaginal and $\mathrm{C}$-section births. However, neither gestational age nor maternal age showed to influence maternal microbiota (data not shown). 
We observed significant correlations between the maternal weight gain during pregnancy and some nutrients consumption (Supplementary file 2). Weight gain over gestation was positively correlated with lipid intake (rho $=0.24, p=0.041$ ) and negatively associated with fibre (rho $=-0.28, p=0.016$ ) and proteins from a vegetable source (rho $=0-0.25, p=0.035$ ).

Table 1. Characteristics of study participants at delivery

\begin{tabular}{|c|c|c|c|c|}
\hline & $\begin{array}{c}\text { Total } \\
(\mathrm{n}=73)\end{array}$ & $\begin{array}{l}\text { Vaginal delivery } \\
\quad(n=45)\end{array}$ & $\begin{array}{l}\text { C-section } \\
(\mathrm{n}=28)^{\mathrm{a}}\end{array}$ & $\mathrm{p}$-value \\
\hline \multicolumn{5}{|l|}{ Maternal Data } \\
\hline Age (years) & $35[31-36.25]$ & $34[31-36]$ & 35 [33-37] & 0.107 \\
\hline Pre-pregnancy BMI $\left(\mathrm{Kg} / \mathrm{cm}^{2}\right)$ & 21.8 [20.43-24.13] & $21.51[20.43-23.88]$ & $21.89[20.46-24.50]$ & 0.617 \\
\hline Weight gain over pregnancy (Kg) & $12.68 \pm 4.28$ & $12.11 \pm 3.52$ & $13.64 \pm 5.23$ & 0.186 \\
\hline Antibiotic during pregnancy (cases) & $29(39.7 \%)$ & $19(42.2 \%)$ & $10(35.7 \%)$ & 0.581 \\
\hline Daily Energy intake (kcal) & $2882 \pm 789$ & $2937 \pm 793$ & $2795 \pm 788$ & 0.458 \\
\hline \multicolumn{5}{|l|}{ Neonate data } \\
\hline Gestational age (weeks) & $40[39-40]$ & $40[39-40]$ & $39[38-40]$ & $0.018^{*}$ \\
\hline Neonatal weight at birth (g) & $3323 \pm 416$ & $3297 \pm 339$ & $3367 \pm 524$ & 0.536 \\
\hline Neonatal height at birth $(\mathrm{cm})$ & $50.16 \pm 1.94$ & $50.12 \pm 1.63$ & $50.24 \pm 2.40$ & 0.821 \\
\hline BMI z-score at birth & $-0.16[-0.60-0.50]$ & $-0.28[-0.66-0.30]$ & $-0.09[-0.48-0.62]$ & 0.314 \\
\hline $\begin{array}{l}\text { Intrapartum antibiotic exposure }\left(\mathrm{n}^{\circ}\right. \\
\text { cases) }\end{array}$ & $35(47.9 \%)$ & $7(15.6 \%)$ & $28(100 \%)$ & $<0.001 *$ \\
\hline Sex & & & & \\
\hline Male & $40(54.8 \%)^{b}$ & $22(48.8 \%)$ & $18(64.3 \%)$ & 0.199 \\
\hline Female & $33(45.2 \%)$ & $23(51.2 \%)$ & $10(35.7 \%)$ & \\
\hline
\end{tabular}

BMI-z scores were computed using WHO Anthro software (www.who.int/ childgrowth/software/en/). Significant categories were marked in bold. (a) One participant in the CS group with missing delivery associated data. Parametric data were expressed as mean $\pm \mathrm{SD}$ and non-parametric data as median [IQR].

\section{Relation between maternal diet and maternal gut microbiota composition}

The maternal diet was presented as a daily intake for each nutrient (Table 2). The energy intake average was 2,882 $\pm 789 \mathrm{kcal} /$ day, without differences according to mode of delivery (vaginal vs C-section). Higher lipid and saturated fatty acids (SFA) consumption during pregnancy was significantly associated with higher animal protein intake and with lower vegetable protein, total carbohydrates, polysaccharides, and fibre (Supplementary file 3). However, diets characterized by higher total carbohydrate, including polysaccharides, consumption during pregnancy were related to a higher 
intake of vegetable protein and fibre. Therefore, we observed two dietary patterns in our population. The first one was characterized by a high intake of lipids, SFA, and animal protein and by a low intake of vegetable protein and fibre; while the second one was based on higher intakes of total carbohydrate, polysaccharide, and fibre and lower intakes of lipids and SFA.

Table 2. Maternal diet during gestation according to mode of delivery

\begin{tabular}{|c|c|c|c|c|}
\hline Diet & Total & Vaginal & C-section & p-value \\
\hline Total protein (g/day) & $120 \pm 40(16.7)$ & $123 \pm 41(16.7)$ & $116 \pm 39(16.7)$ & 0.477 \\
\hline Animal protein (g/day) & $80 \pm 33(11.1)$ & $81 \pm 33(11.0)$ & $79 \pm 35(11.3)$ & 0.820 \\
\hline Vegetable protein (g/day) & $40 \pm 3(5.6)$ & $42 \pm 13(5.8)$ & $37 \pm 13(5.3)$ & 0.817 \\
\hline Lipids (g/day) & $143 \pm 45(44.5)$ & $142 \pm 43(43.4)$ & $144 \pm 49(46.4)$ & 0.814 \\
\hline SFA (g/day) & $40 \pm 16(12.2)$ & $39 \pm 16(11.9)$ & $40 \pm 18(12.7)$ & 0.963 \\
\hline MUFA (g/day) & $67 \pm 20(21.3)$ & $67 \pm 21(20.9)$ & $67 \pm 20(22.0)$ & 0.502 \\
\hline PUFA (g/day) & $25 \pm 12(7.7)$ & $24 \pm 10(7.36)$ & $26 \pm 15(8.3)$ & 0.568 \\
\hline Cholesterol (mg/day) & $422 \pm 170$ & $431 \pm 170$ & $408 \pm 173$ & 0.106 \\
\hline Carbohydrates (g/day) & $277 \pm 88(38.5)$ & $291 \pm 86(39.6)$ & $256 \pm 87(36.7)$ & 0.168 \\
\hline Polysaccharides (g/day) & $142 \pm 47(19.8)$ & $148 \pm 47(20.3)$ & $131 \pm 74(19.1)$ & 0.148 \\
\hline Fibre (g/day) & $36 \pm 14(2.5)$ & $38 \pm 15(2.7)$ & $32 \pm 11(2.3)$ & 0.084 \\
\hline Vitamin A (mcg/day) & $1647 \pm 863$ & $1659 \pm 896$ & $1629 \pm 824$ & 0.887 \\
\hline Retinoid (mcg/day) & $393 \pm 245$ & $410 \pm 264$ & $366 \pm 212$ & 0.453 \\
\hline Carotenoid (mgc/day) & $7517 \pm 4642$ & $7482 \pm 4900$ & $7572 \pm 4282$ & 0.937 \\
\hline Vitamin D (mcg/day) & $3 \pm 2$ & $4 \pm 3$ & $3 \pm 2$ & 0.156 \\
\hline Vitamin E (mg/day) & $22 \pm 9$ & $22 \pm 7$ & $23 \pm 11$ & 0.604 \\
\hline Thiamine (mg/day) & $2 \pm 0.7$ & $3 \pm 0.8$ & $2 \pm 0.6$ & 0.507 \\
\hline Riboflavin (mg/day) & $2 \pm 0.8$ & $3 \pm 0.9$ & $2 \pm 0.7$ & 0.470 \\
\hline Niacin (mg/day) & $28 \pm 9$ & $29 \pm 10$ & $27 \pm 8$ & 0.331 \\
\hline Vitamin B6 (mg/day) & $3 \pm 1.2$ & $4 \pm 1.4$ & $3 \pm 0.9$ & 0.208 \\
\hline Folic acid (mcg/day) & $653 \pm 282$ & $672 \pm 315$ & $623 \pm 221$ & 0.478 \\
\hline Vitamin B12 (mcg/day) & $10 \pm 8$ & $10 \pm 8$ & $10 \pm 8$ & 0.955 \\
\hline Vitamin C (mg/day) & $347 \pm 168$ & $356 \pm 190$ & $333 \pm 125$ & 0.573 \\
\hline
\end{tabular}

Each nutrients value was extracted by the food frequency questionnaire using the Centro de Enseñanza Superior de Nutrición Humana y Dietética (CESNID) tables [494]. The percentage of caloric contribution of each nutrient to total energy intake was presented in brackets. Saturated fatty acids (SFA), monounsaturated fatty acids (MUFA), polyunsaturated fatty acids (PUFA).

Maternal diet during gestation was associated with the maternal microbiota composition (Fig 1). In the multivariate analysis, the results showed that MUFA intake significantly affects the overall structure of maternal microbiota at delivery $(p=0.03$, PERMANOVA) at the ASV level (Fig 1, A). We found a positive correlation between lipid intake and Firmicutes phylum $($ rho $=0.30, p=0.014)$. Mothers classified as having 
high lipids $(p<0.001)$ and consumers of SFA $(p=0.011)$ had lower Proteobacteria relative abundance in their microbiota (Fig 1, B-E). However, mothers classified as having higher carbohydrate consumption showed a microbiota enriched by this phylum ( $p=0.003$ ). A similar pattern was found with Bacteroidetes phylum, which was found to be enriched in microbiota of mothers with higher carbohydrates intake $(p=0.052)$ and depleted in lipids consumers $(p=0.011)$. Besides this relation, total protein intake, especially those from an animal source (rho $=-0.32, p=0.009$ ), was negatively associated with Actinobacteria phylum genera.

At the genus level, several correlations were found between nutrient intake and maternal microbiota (Supplementary file 4); a higher relative abundance of Lachnospira was associated with lower total carbohydrates intake (rho $=-0.27, p=$ 0.040 ) and showed a positive correlation with total lipid intake (rho $=0.28, p=0.039$ ), including SFA and MUFA but not PUFA. Similarly, a pattern was found in Rombustia genus, which was associated with MUFA intake (rho $=0.28, p=0.025$ ) but negatively linked with total carbohydrates (rho $=-0.25, p=0.049)$ intake, especially polysaccharides (rho $=-0.28, p=0.22)$. No significant effect of any nutrient consumption was observed in the alpha diversity of maternal microbiota at delivery. 


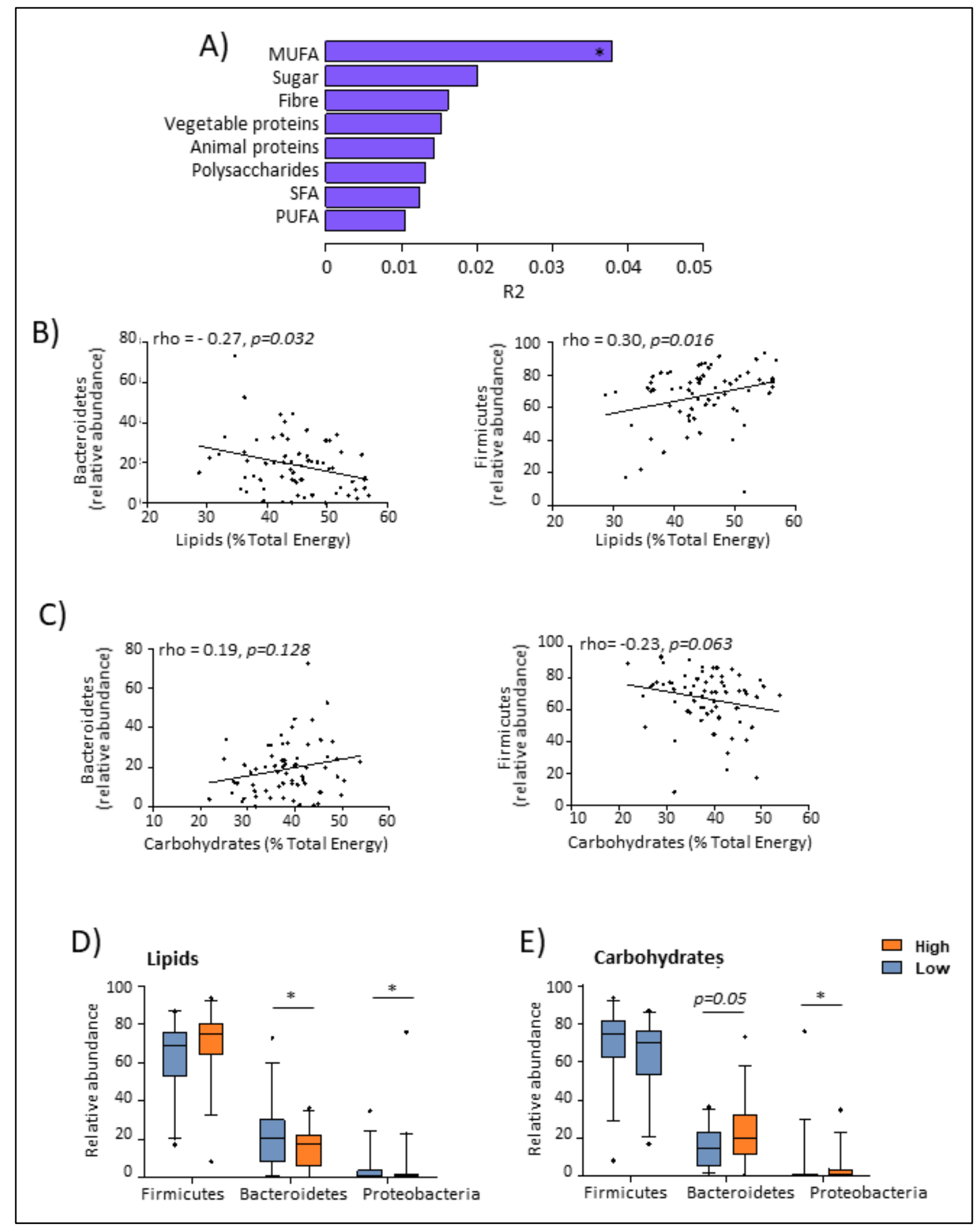

Figure 1. Maternal diet was associated with maternal microbiota. A) Multivariate analysis of the effect of studied nutrients on maternal microbiota composition at delivery based on Bray-Curtis. B) Maternal lipids intake was associated to the relative abundances of Bacteroidetes and Firmicutes (Spearman's rank correlation). C) Maternal total carbohydrates intake was associated to maternal relative abundances of Bacteroidetes and Firmicutes (Spearman's rank correlation). D-E) Comparison of principal phyla of maternal microbiota between mothers classified as high/low consumers of total lipids (D) and carbohydrates intakes (E). Nutrients consumption was expressed as caloric contribution to total energy intake in percentage. 


\section{Maternal diet and intestinal markers}

Maternal diet was associated with the intestinal zonulin concentrations (42.05 \pm $9.9 \mathrm{ng} / \mathrm{ml}$ faecal supernatant) at birth. A lower zonulin concentration was related to a higher total carbohydrate intake (rho $=-0.37, p=0.012$ ), including polysaccharide (rho $=-0.31, p=0.035$ ) intake during pregnancy. However, a higher consumption of lipids (rho $=0.32, p=0.031)$, including MUFA intake (rho $=0.29, p=0.05)$, was associated with higher zonulin concentrations (Fig 2, A-D). Neither protein nor fibre consumption presented any significant correlation with zonulin concentration on maternal stool samples.

We found some positive relations between excreted zonulin concentrations and some genus of maternal microbiota. A higher zonulin concentration showed a tendency to lower Proteobacteria phylum (rho $=-0.28, p=0.067$ ). Zonulin was positively correlated with relative Rombustia (rho $=0.32, p=0.033$ ) and Turicibacter (rho $=0.31$, $p=0.041)$ genera.

Furthermore, higher zonulin concentrations were significantly associated ( $p=$ 0.021) with higher intestinal alkaline phosphatase (IAP) activity, considered as a measure of intestinal function $(0.06$ [0.03 - 0.21] $\mathrm{U} / \mathrm{ml}$ faecal supernatant) (data not shown). We also found a positive correlation between IAP concentration and excreted zonulin (rho $=0.349, p=0.019$ ). Although no significant correlations were observed between IAP activity and any nutrient intake, IAP activity was observed to be related to some genera of maternal microbiota (Supplementary file 4). Similarly to the zonulin associations, IAP was also positively linked to some Firmicutes members, including the genus Rombustia (rho $=0.31, p=0.031$ ) and Turicibacter (rho $=0.35, p=0.015$, but also some Ruminococcaceae groups including Ruminococcaceae_UCG013 (rho $=0.39$, $p=0.006$ ), Roseburia (rho $=0.37, p=0.009)$, and Intestinibacter $($ rho $=0.38, p=0.007$ ). Additionally, lower abundance of Proteobacteria phylum was associated with higher IAP activity ( $r$ ro $=-0.27, p=0.062$ ).

Intestinal inflammation, measured by calprotectin concentrations $(10.73$ $[\mathrm{IQR}=2.38-20.71] \mu \mathrm{g} / \mathrm{g}$ ), was not found to be related to maternal nutrient intake during pregnancy. Calprotectin faecal concentrations were also not affected by the mode of delivery $(p=0.084)$ and intrapartum antibiotic exposure $(p=0.668)$. Moreover, calprotectin concentration was negatively associated with Rombustia genus (rho = $0.32, p=0.029)$, that it was also related to zonulin and IAP concentrations. 


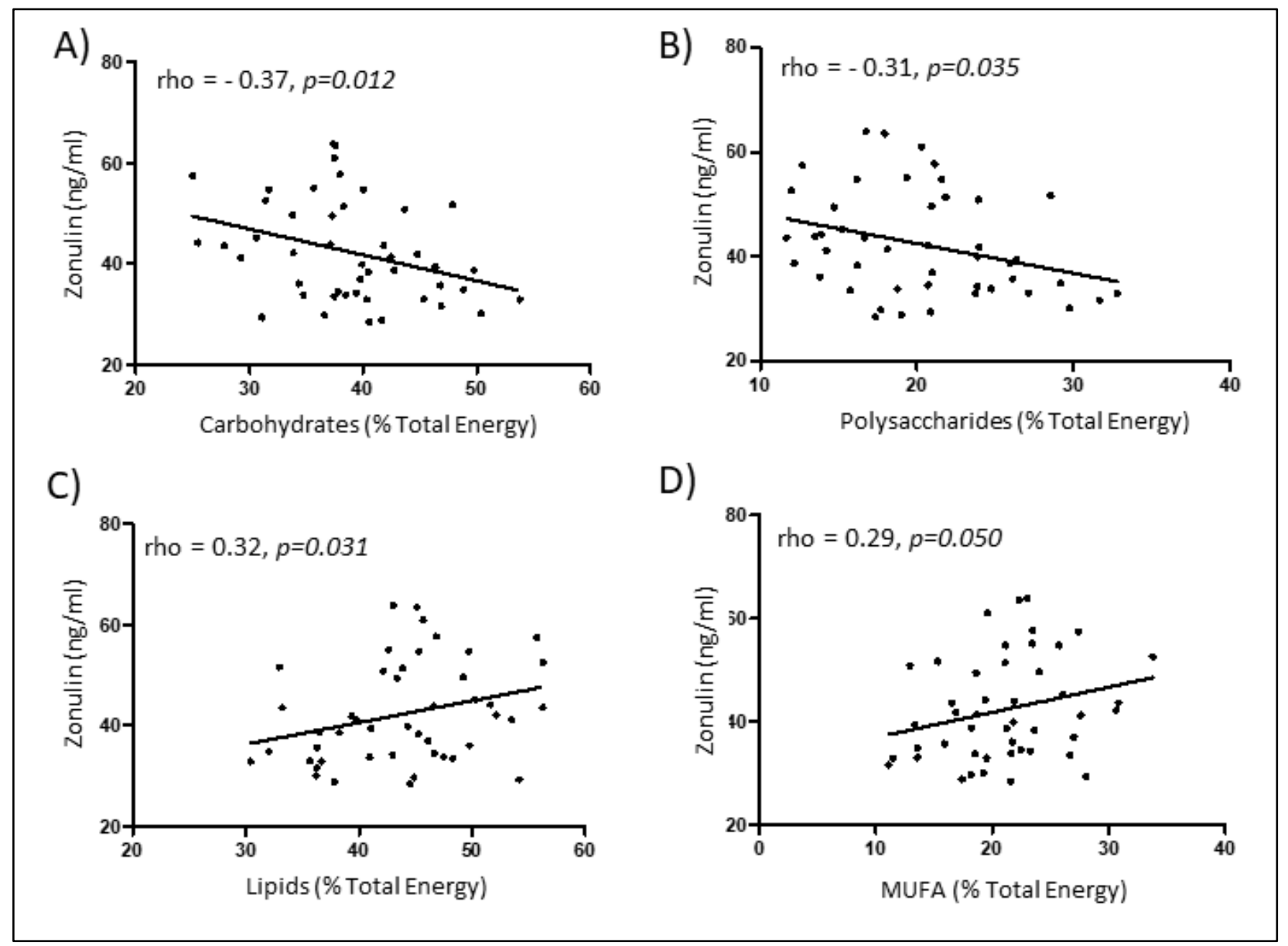

Figure 2. Maternal diet was associated with intestinal markers. Spearman's rank correlation between total energy contribution (in percentage) of total carbohydrates (A), polysaccharides (B), lipids (C) and MUFA (D) and zonulin concentration in maternal stool samples.

Relation between maternal diet, intestinal markers, and neonatal gut microbiota composition in a birth-dependent manner

A significant difference was found between the composition of maternal and infant microbiota (Supplementary file 5). Neonatal microbiota was characterized by lower alpha-diversity, measured using the Chao indices $(p<0.001)$ and Shannon index $(p=0.06)$, and a significant enrichment in genera from Proteobacteria (18.9\% vs. $0.8 \%$ in neonates and mothers, respectively, $p<0.001)$ and Actinobacteria $(15.4 \%$ vs. $4.07 \%$ in neonates and mothers, respectively, $p<0.001$ ) phylum compared to maternal microbiota.

In terms of alpha-diversity, the results showed that maternal diet during gestation was associated with the diversity and richness of neonatal microbiota (Fig 3). 
Neonatal microbiota richness, measured using the Chao index, was positively correlated to SFA (rho $=0.24, p=0.047)$ and proteins from animal sources $($ rho $=0.25, p=0.038$ ) and negatively associated with maternal vegetable protein intake (rho $=-0.25, p=$ 0.045). Similarly, the neonatal bacterial diversity measured by Shannon index was negatively correlated to vegetable proteins (rho $=-0.30, p=0.013)$ and fibre (rho = $0.26, p=0.037)$ intakes.

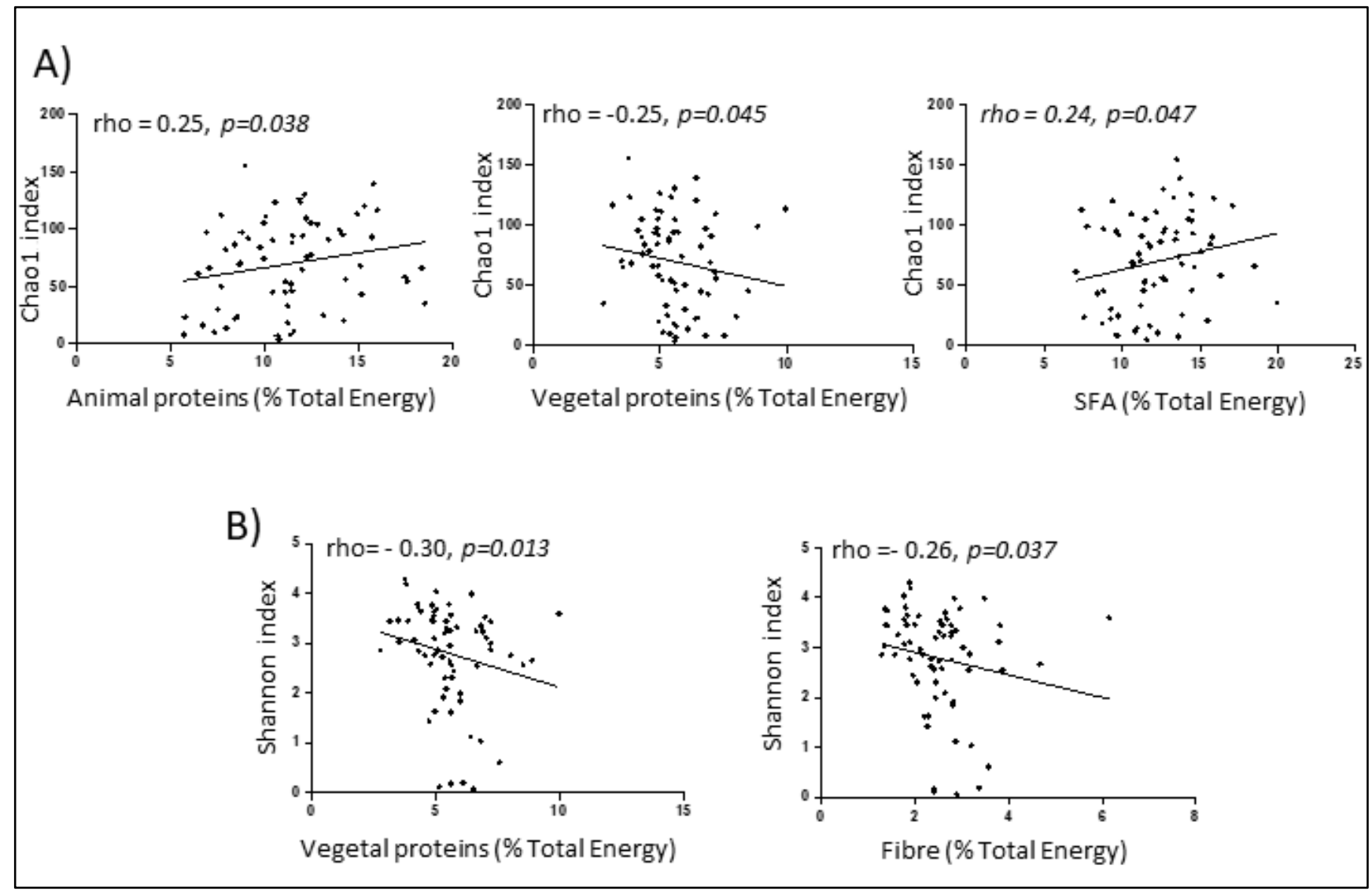

Figure 3. Maternal diet was associated with the neonatal microbial richness and diversity. A) Significant associations (Spearman's rank correlation) between neonatal microbial richness (measured as Chao index) and maternal intake of proteins from animal and vegetable sources and saturated fatty acids (SFA). B) Correlations between vegetal proteins and fibre consumption and neonatal microbiota diversity measured by Shannon index.

Specific neonatal bacterial groups were associated with nutrient patterns from the maternal diet. At the phylum level, we observed that fat-related nutrients intake by mothers, including total lipids, SFA, and MUFA, but not PUFA, showed enrichment in Firmicutes phylum genera and a depletion in Proteobacteria phylum genera in the offspring microbiota (Supplementary file 7-8). Thus, a negative correlation was found 
between the total lipids ( $r h o=0.27, p=0.026)$, SFA ( $\mathrm{rho}=0.29, p=0.016)$, MUFA (rho $=0.32, p=0.008$ ), and Proteobacteria phylum.

At the genus level (Fig 4), a cluster composed of several vitamins, proteins from plant-based sources, and fibre was negatively correlated with the relative abundance of the genus Coprococcus, Blautia, Roseburia, and several groups from the Ruminococcaceae and Lachnospiraceae families in neonatal faecal samples. Most of these genera were enriched and had a positive correlation with the maternal intake of lipids, MUFA, and animal protein, being especially significant the maternal intake of SFA. The only link of maternal zonulin and neonatal gut microbiota was found for Bacteroidetes phylum. Higher maternal zonulin was associated with a higher relative abundance of Bacteroidetes phylum $(p=0.042)$, including ASVs from the Bacteroides $(p=0.019)$ genus. 


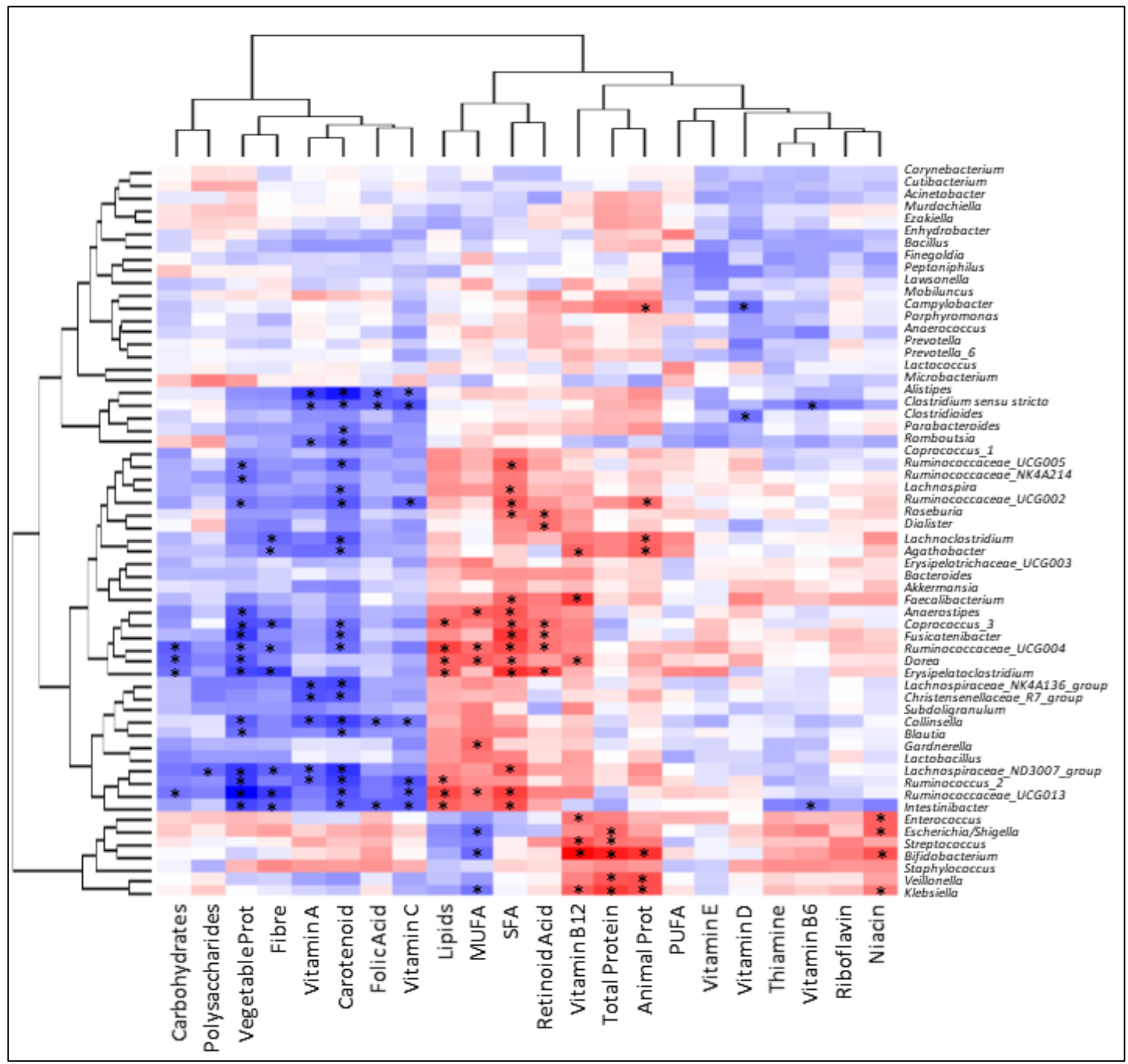

Figure 4. Maternal diet was associated with neonatal microbiota at birth. Spearman correlations between maternal nutrients intake during pregnancy and neonatal bacterial at genus level at birth. Significant correlations $(p<0.05)$ were marked by an asterisk (*). Red colour represents the positive correlations whereas blue colour shown negative relations. The most abundant genera were showed in the graph. Bacterial genera and dietary components are grouped at the square edges

When stratifying the infants according to birth mode (vaginal or C-section) and the maternal intake of total protein $(p=0.001)$, plant-based protein $(p=0.001)$, lipids $(p$ $=0.006)$, total carbohydrates $(p=0.007)$, and fibre $(p=0.003)$, we observed that these nutrients have different effects on neonatal microbiota according to the mode of 
delivery (Supplementary file 6). Further, the maternal intake of SFA and fibre reduced the microbiota shifts related to delivery mode (Fig 5). The Bray-Curtis similarity index between vaginal and C-section born infants was lower in offspring from mothers who had higher intakes of SFA $(p<0.001)$ and fibre $(p<0.001)$ (Fig 5, C-D).

A)

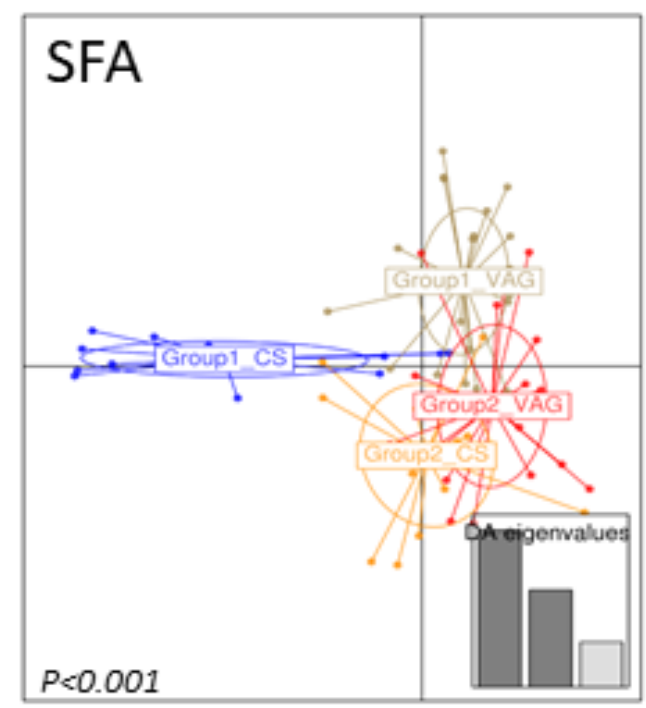

C)

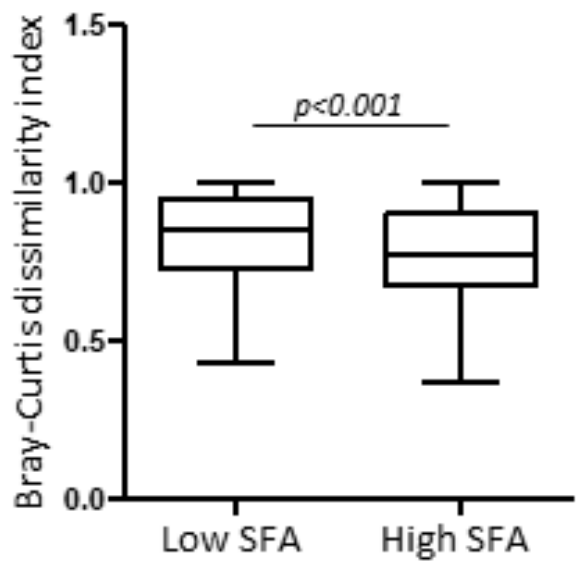

B)

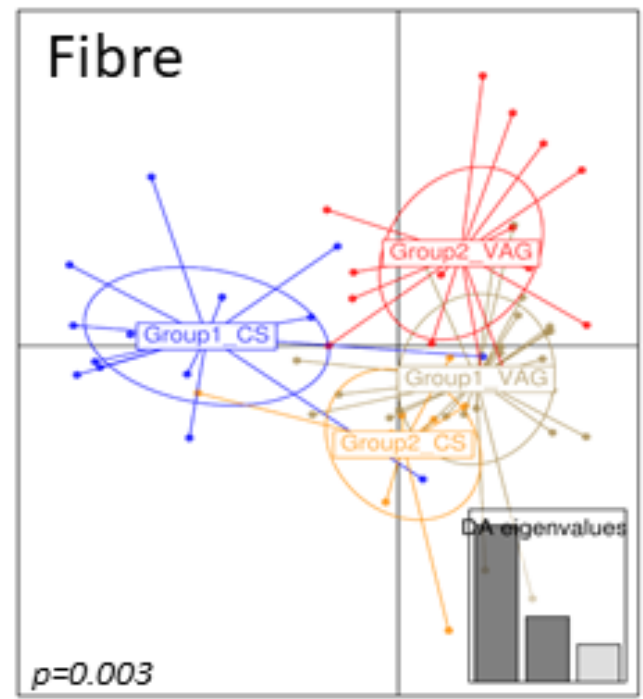

D)

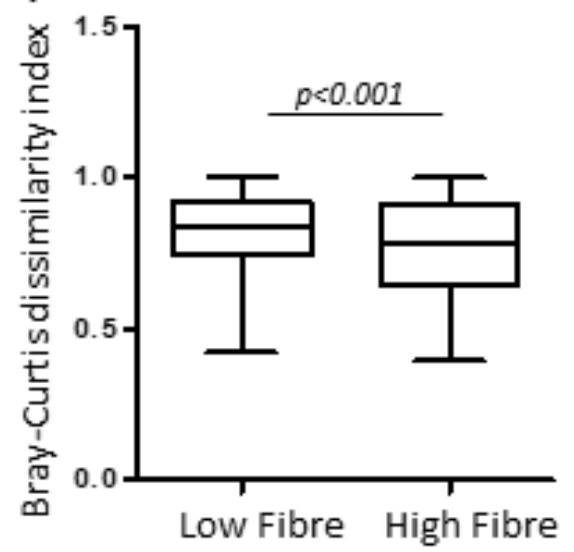

Figure 5. Neonatal microbiota was distinctly affected depending on mode of birth and maternal diet. Discriminant Analysis of Principal Components (DAPC) of the neonatal microbiota according to mode of delivery and maternal nutrients intake classification. VAG (Vaginal delivery), CS (C-Section), Group1/2: maternal consumption of the each nutrient lower (1)/higher (2) than average intake of the study population, including saturated fatty acids (A) and fibre (B). Bray-Curtis dissimilarity index within both vaginal and C-section born infants according to their mothers had high or low consumption of SFA (C) and fibre (D). 
In general, we observed a higher effect of maternal diet in vaginal delivered infants than CS-born neonates, especially of fat-related nutrients and vegetable source proteins and fibre. The positive correlations were maintained in the independent analysis of the vaginal delivered neonates (Supplementary file 7). Total lipids were negatively linked to Escherichia/Shigella genus (rho $=-0.38, p=0.014$ ) and positively associated with Firmicutes phylum, including genera from the Ruminococcaceae groups and from the Blautia, Roseburia, Rombustia, and Faecalibacterium genera. Similarly, these genera also showed a negative correlation with fibre and vegetable source proteins. The total carbohydrates (rho $=-0.34, p=0.028$ ), vegetable source proteins (rho $=-0.31, p=0.046$ ), and fibre (rho $=-0.30, p=0.054$ ) were negatively linked with Firmicutes phylum genera. Similarly, we observed a lower relative abundance of Proteobacteria phylum in the maternal microbiota SFA $(p<0.001)$ and MUFA $(p$ $=0.012)$ intake.

However, in the CS-born infants (Supplementary file 8), we found less relation between maternal diet and neonatal microbiota. Thus, we observed that the offspring of mothers classified as higher fibre consumers showed a higher relative abundance of Proteobacteria phylum genera $(p=0.046)$. Similarly, the maternal PUFA intake was positively correlated with this phylum (rho $=0.44, p=0.028)$ and negatively associated with Firmicutes phylum $($ rho $=0.40, p=0.046)$.

At the genus level, we found correlations of neonatal microbiota and maternal protein intake, especially those from animal sources, including Veillonella (rho $=0.54$, $p=0.005)$, Escherichia/Shigella $($ rho $=0.40, p=0.045)$, Klebsiella $(\mathrm{rho}=0.43, p$ $=0.032)$, or Clostridium sensu stricto $($ rho $=0.40, p=0.047)$ genera.

\section{DISCUSSION}

This study shows that maternal diet during pregnancy is associated with the maternal intestinal markers, including gut microbiota, and the offspring microbiota composition at delivery.

It is well known that diet is a main driver of the human gut microbiome that can modulate state of health. Pregnancy is a sensitive period related to the health programming of offspring [546,547]. Recently, a systematic review found associations between the maternal diet and the maternal microbiota (five papers) or infant gut 
microbiome (two papers) [198], but no studies have examined both maternal and infant microbiota simultaneously. In our study, the mothers followed a conventional healthy diet, without any intervention, and they were not obese or diabetic. The maternal consumption of different nutrients showed a homogenous pattern and was in concordance with other studies performed on a Spanish general population $[548,549]$ and on pregnant women [550]. Therefore, the associations between nutrients and maternal or infant gut microbiota are likely caused by subtle changes in nutritional patterns, which better represents the general population than extreme diets (e.g., high fat vs. high fibre).

We found relations between maternal microbiota and specific nutrients, such as fat-related nutrients intake and Firmicutes phylum, including Lachnospira genus and a reduction of Proteobacteria phylum. Several studies have shown the increase in the relative abundance of Firmicutes in intestinal microbiota with increased body mass index [237] and in response to a high fat diet [551]. Furthermore, Koren et al. found that maternal microbiota was altered during late pregnancy, increasing the Proteobacteria and Actinobacteria phyla, which contribute with a positive feedback loop to host adiposity and a low grade of inflammation commonly observed in normal pregnancy development [132]. Our results suggest that maternal diet could modify the progress of microbiota remodelling that has been observed during pregnancy and affect the motherinfant transmission.

Several studies have shown how maternal diet determines the variations of infant microbiota [198]. In a primate model, the maintenance of a high-fat diet through pregnancy resulted in a significant variation in intestinal commensal taxa in the offspring, with an enrichment of Ruminoccoccus and Dialister genera and a reduction of the Campylobacter genus [193]. These results were partially confirmed by the same authors in humans [194]. Infants born from mothers with a higher fat consumption during gestation had a relative depletion of the Bacteroides genus in their gut microbiota, observable up to six weeks [194]. In 2019, Lundgren et al. observed that maternal diet had an effect on infant gut microbiota that could differ by delivery mode [195]. Mandal et al. reported that fat and some fat-soluble vitamins influenced the maternal gut microbiota changes during pregnancy [196]. Notably, we found a cluster of vitamins with a significant association with neonatal microbiota, including vitamins $D$, A, and B12. In 2019, Boran et al. observed positive correlations between vitamin B12 status and different genera of Firmicutes phylum, while negative associations were 
reported for Clostridiales and Prevotella, among others, in infants' bacterial gut population [197]. However, the effect of these relations and whether they are cause or consequence of the microbiota alterations observed remain unclear. Therefore, the link between maternal diet during pregnancy and gut microbiota may be involved in maternal intestinal metabolic function and in the vertical transmission of specific genera impacting early neonatal colonization.

Studies have shown that maternal intestinal permeability is affected during gestation. Pregnant woman had higher permeability compared to non-pregnant woman [552]. It is unknown whether this could have implications for maternal-infant microbial transference. In our study, lipids, total carbohydrates, and polysaccharides showed the greatest influence on the maternal intestinal function, assessed by faecal zonulin, calprotectin, and IAP. These markers of inflammation and functional maturation $[308,540,553-556]$ have been previously reported as responsive to dietary interventions in the human population [557].

It is recognized that the consumption of excess dietary fats can enhance intestinal permeability [558,559]. Kim et al. showed that high fat diet (HFD) increased endotoxin concentrations in the intestinal lumen and plasma through an altered permeability that leads to systemic inflammation [560]. In our study, zonulin was increased in mothers with higher fat intake. Altered upregulation of zonulin secretion from the intestinal epithelial cells into the lumen can increase paracellular permeability [561,562] and previous research showed that some nutrients, especially those related to fats, can increase faecal zonulin concentrations [557,563]. Although intestinal permeability cannot be directly linked to zonulin levels in faeces, increased faecal zonulin is described in professional athletes [564], Crohn disease patients [565] or individuals with metabolic disorders [308,566], all physiological or pathological conditions related with impaired intestinal homeostasis.

While other studies linked high-fat diets and IAP expression [567], we could not confirm this observation in our data. However, in our population, fat-related nutrients were related to maternal microbiota, thus increasing some Firmicutes genera that are also associated with higher IAP concentrations. Thus, the link fat-zonulin-IAPmicrobiota could be a relation between fat metabolism and maternal microbiota at delivery with possible consequences in the mother-infant transference. We have shown that this link is also observable in first-pass infant microbiota. Most previous studies that focused on IAP were conducted on animals using an experimental design that 
specifies extremely different feeding groups; however, our study did not permit the control of all variables affecting IAP expression. The faecal concentration of calprotectin in the mothers of our study was in the range of the reported values in other studies [554]. Our results suggested that the link of diet on maternal faecal zonulin and IAP is not related to an increment of inflammatory status in the gut, since no differences were observed in terms of calprotectin concentration. In that sense, one weakness of our study is the lack of data on intestinal markers in the offspring, which would allow for establishing associations of maternal nutrients and effects on infant permeability and will ensure further research.

Beyond maternal shifts in microbiota, gestational diet and intestinal functionality may be associated with offspring microbiome shifts, especially induced by lipid intake. Based on previous research, pre-natal exposure to bacterial metabolites (e.g., short chain fatty acids), endotoxins or lipopolysaccharide, and actual bacteria may occur in healthy pregnancies, tightly regulated by intestinal permeability (reviewed in Walker et al. in 2017) [568]. Lipids, especially SFA and MUFA, and fibre had a significant impact on neonatal microbiota. Neonates born from mothers with a higher fat consumption, including lipids and SFA, showed enrichment of bacterial taxa from Firmicutes phylum-such as Lachnospira, Roseburia, and Coprococcus genera, and some Ruminococcaceae groups - and a depletion of other taxa typically found on neonatal meconium, especially those from Proteobacteria phylum such as Escherichia/Shigella genus. Shifts on microbiome acquisition may have unknown consequences for priming the immune system during a critical window of immune development.

The effect of maternal diet on infant microbiome structure was more evident in vaginally delivered infants than in those born by C-Section, possibly influenced by labour and birth-related factors affecting microbiome transference (e.g., contact with vaginal and faecal microbiota, hormonal cascades [130,153], pre-surgical intervention fasting [569]). C-section born infants have less contact with maternal gut microbiota during delivery and are inevitably exposed to antibiotics at birth [163,164], which could modify the effect of perinatal factors, such as maternal diet. Since almost all of Csections in our study were elective, the mothers did not have any labour process. The Csection procedure is associated with antibiotic administration; therefore, we cannot discriminate between the effects of antibiotics or the method of birth, and we consider both as concomitant factors affecting the microbial transmission from the mother to the neonate. 
Remarkably, we analysed $16 \mathrm{~S}$ configurations at delivery, which may not necessarily correlate well with the $16 \mathrm{~S}$ configurations that rapidly establish themselves very early in life. However, growing evidence suggested that the composition of the meconium or first-pass faeces microbiota could have a relevant influence in the colonization process in the later days and months, but also in the immune priming and epigenetic programming of the foetus and neonate [570].

Among the limitations of the study, FFQ-Dietary information is subject to memory bias as well as the lack of perception on the food proportion sizes. Therefore, the use of specific $24 \mathrm{~h}$ recall and/or 3 days recall questionnaires including portions would be needed for future studies. Furthermore, our sample size was limited and further studies with higher number of participants and also, with the follow-up of the participants would be needed. Despite all those limitations, our study demonstrated how maternal diet during pregnancy, especially related to lipid intake, had a significant effect on maternal intestinal function and neonatal microbiome, potentially contributing to an adequate transmission of initial colonization and health-programming. Therefore, specific dietary programs targeting pregnant women may be a cost-effective intervention factor to promote adequate vertical mother-infant exposition.

\section{CONCLUSION}

Fat intake during pregnancy is associated with maternal microbiome and intestinal permeability, which could affect the initial microbial exposure and acquisition of the neonate. The importance of the priority events on the interplay host-microbiome is crucial. Our results shed light on the unexplored mechanisms, whereby maternal diet can influence the neonatal development and highlight the crucial role of maternal diet in infant colonization. These findings highlight the importance of dietary counselling for a correct mother-infant microbial transmission. 


\section{SUPPLEMENTARY DATA}

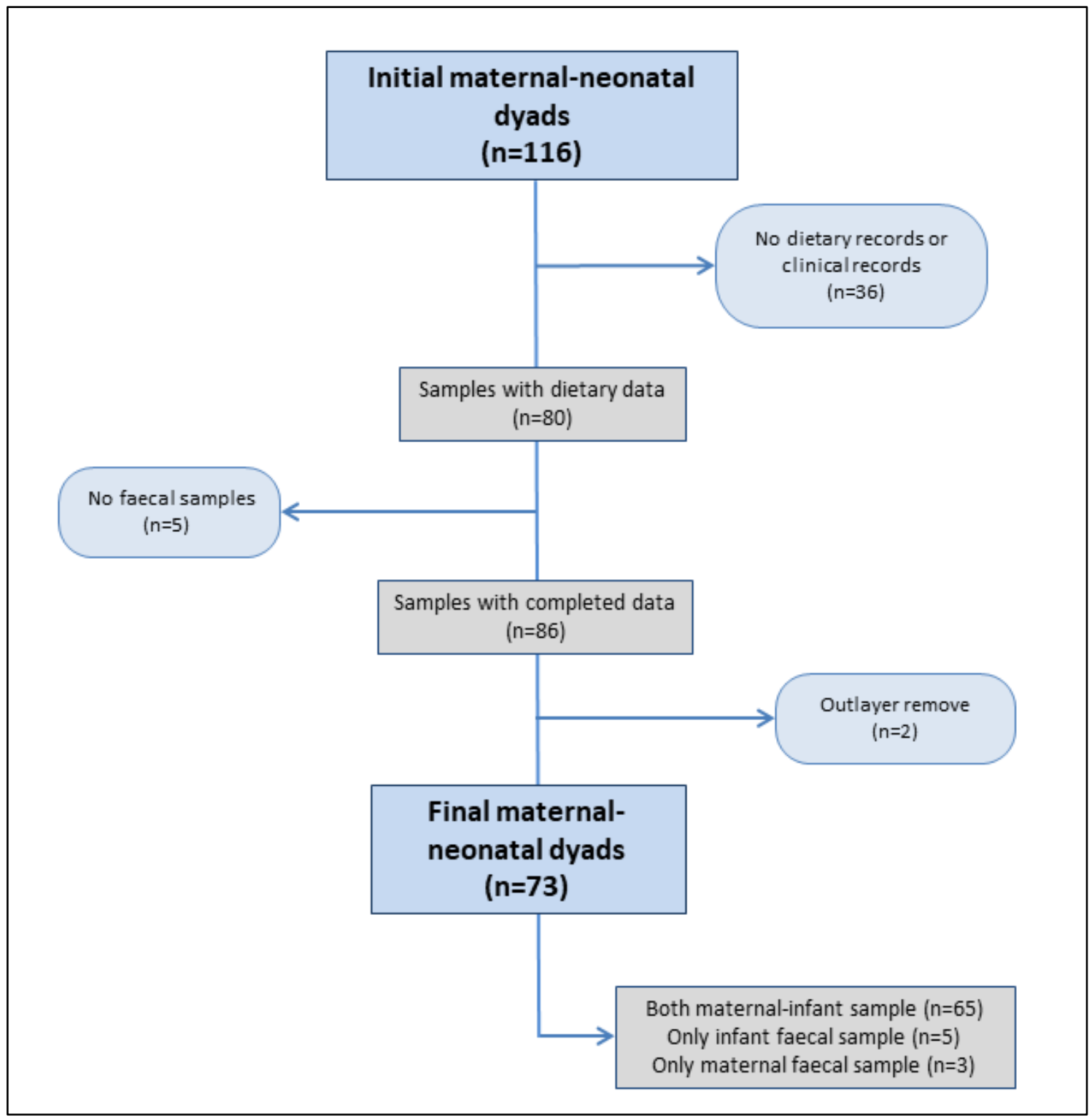

Supplementary file 1. Flow-chart of participants in the study 


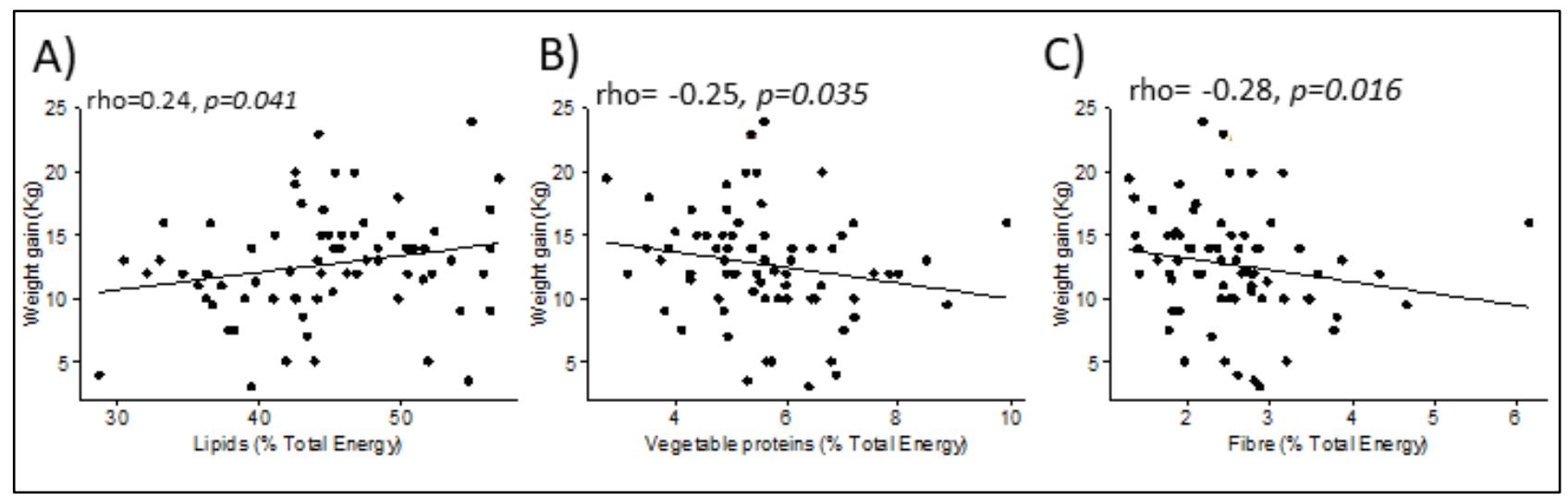

Supplementary file 2. Maternal diet was related to the weight gain during pregnancy. Spearman correlation between total energy contribution (in percentage) of total lipids intake (A), proteins from vegetable source $(\mathbf{B})$ and fibre $(\mathbf{C})$ and maternal weight gain during pregnancy $(\mathrm{Kg})$. 
Supplementary file 3. Spearman correlations between reported nutrients intake of maternal diet during pregnancy.

\begin{tabular}{|c|c|c|c|}
\hline Nutrient & & rho & p-value \\
\hline Animal pro & & & \\
\hline & $S F A$ & 0.338 & 0.003 \\
\hline & Carbohydrates & -0.461 & $<0.001$ \\
\hline & Polysaccharide & -0.305 & 0.009 \\
\hline Vegetal pro & & & \\
\hline & Lipids & -0.644 & $<0.001$ \\
\hline & $S F A$ & -0.642 & $<0.001$ \\
\hline & $M U F A$ & -0.454 & $<0.001$ \\
\hline & Carbohydrates & 0.547 & $<0.001$ \\
\hline & Polysaccharide & 0.573 & $<0.001$ \\
\hline & Fibre & 0.832 & $<0.001$ \\
\hline Lipids & & & \\
\hline & Carbohydrates & -0.896 & $<0.001$ \\
\hline & Polysaccharides & -0.743 & $<0.001$ \\
\hline & Fibre & -0.533 & $<0.001$ \\
\hline Saturated I & & & \\
\hline & Carbohydrates & -0.647 & $<0.001$ \\
\hline & Polysaccharides & -0.489 & $<0.001$ \\
\hline & Fibre & -0.507 & $<0.001$ \\
\hline Monounsat & tty acids & & \\
\hline & Carbohydrates & -0.623 & $<0.001$ \\
\hline & Polysaccharides & -0.510 & $<0.001$ \\
\hline & Fibre & -0.297 & 0.011 \\
\hline
\end{tabular}

Only macronutrients were included in the table. Saturated (SFA) and mono- (MUFA) unsaturated fatty acids. 


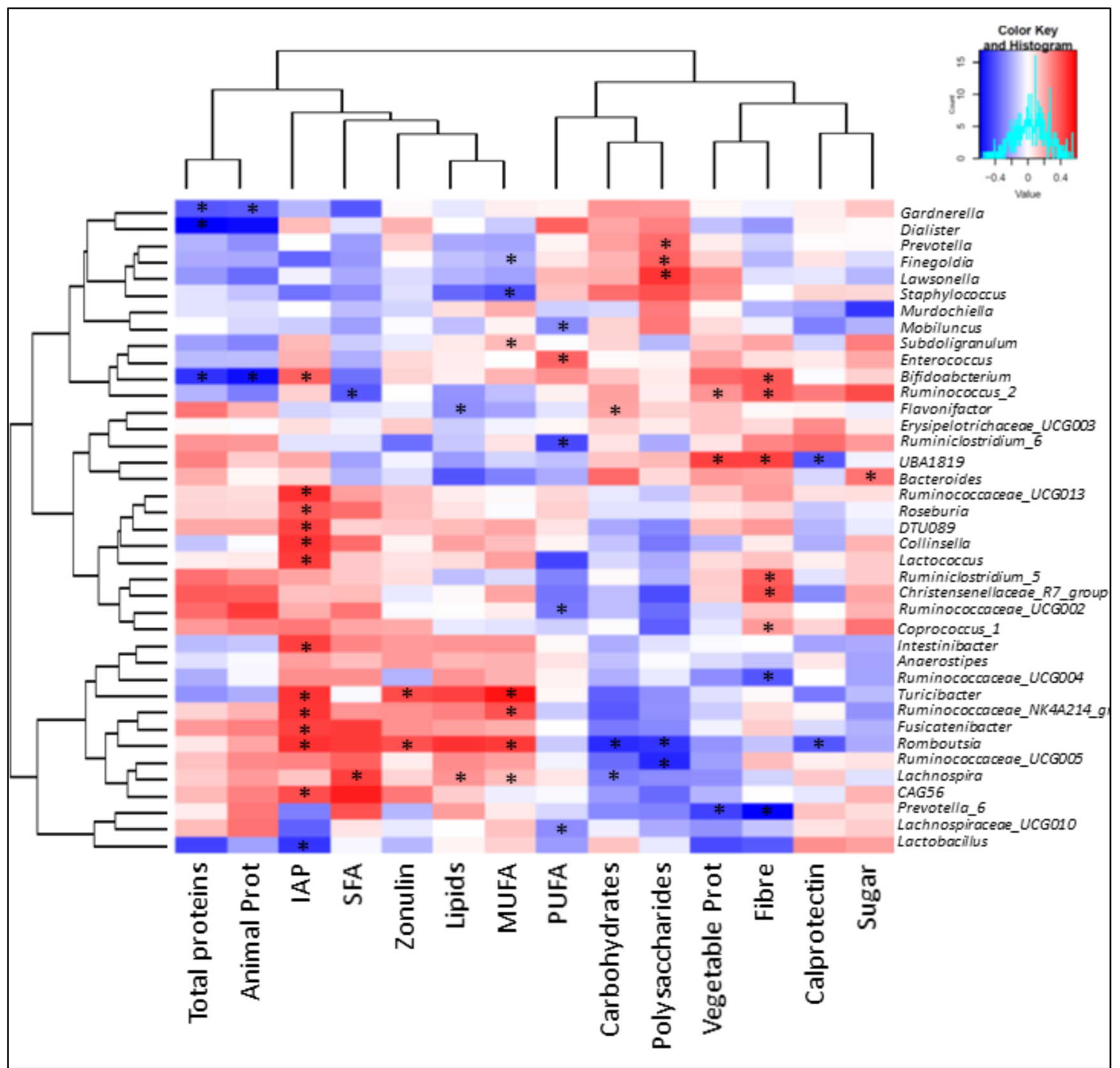

Supplementary file 4. Maternal diet and intestinal markers were associated with the maternal microbiota at delivery. Spearman correlations between maternal nutrients intake during pregnancy and maternal bacterial at genus level at birth. Significant correlations $(p<0.05)$ were marked by an asterisk $\left(^{*}\right)$. Red colour represents the positive correlations whereas blue colour shown negative relations. Bacterial genera and dietary components are grouped at the square edges. Only genera with significant relations were shown. 


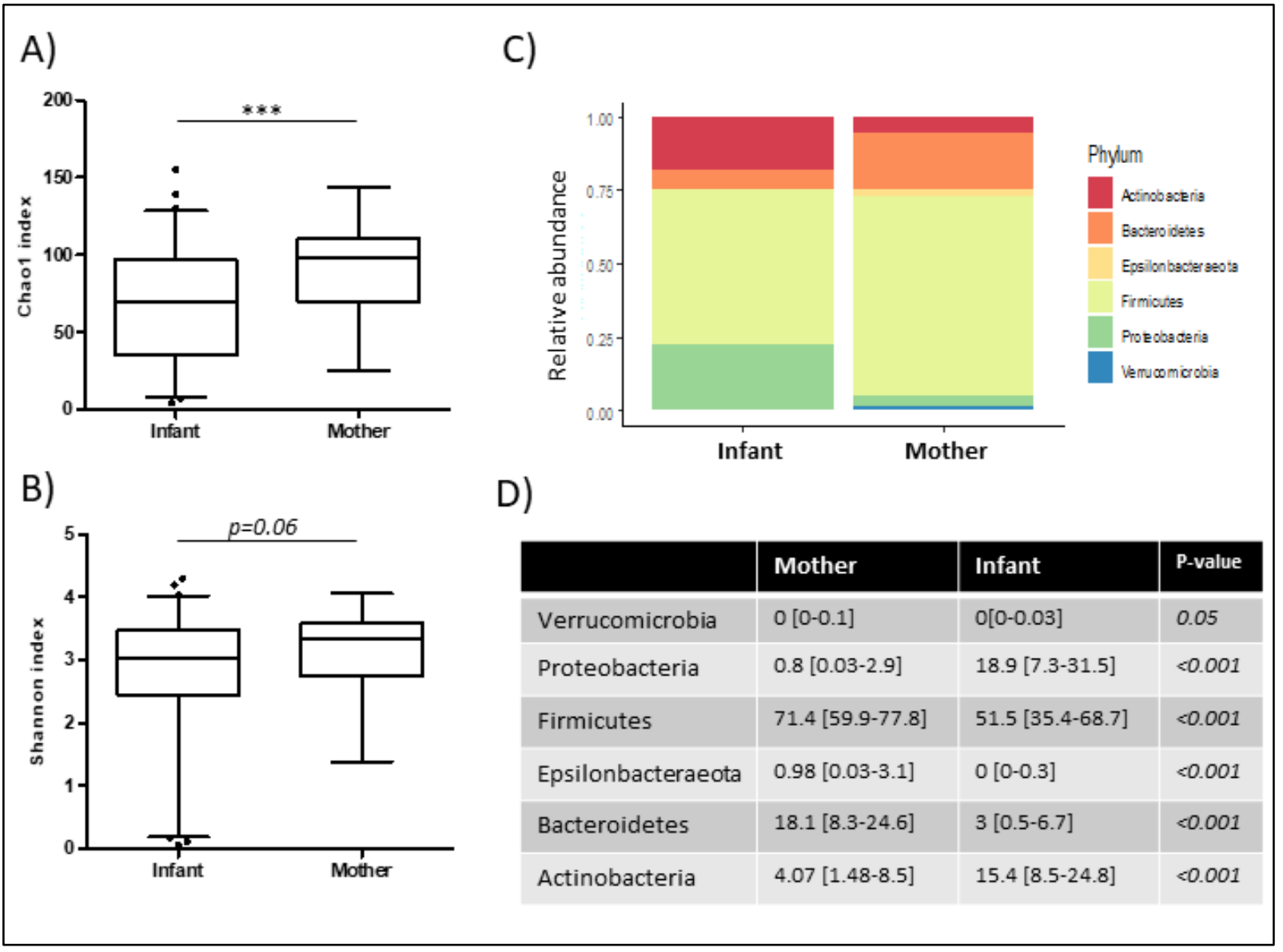

Supplementary file 5. Comparison of bacterial population from maternal intestinal microbiota and infant faecal swabs microbiota. A-B) Alpha diversity measured by Chao1 (A) and Shannon (B) index of infant and maternal microbiota at delivery. C) Bar-plots at phylum level of maternal and neonatal microbiota at delivery. D) Relative abundance of each phylum was expressed as median and interquartile range between brackets. Mann-Whitney test with FDR adjustment was used to test the significance of the differences between maternal stool and infant microbiota and for diversities analysis. * $p<0.05, * * p<0.01, * * * p<0.001$. 


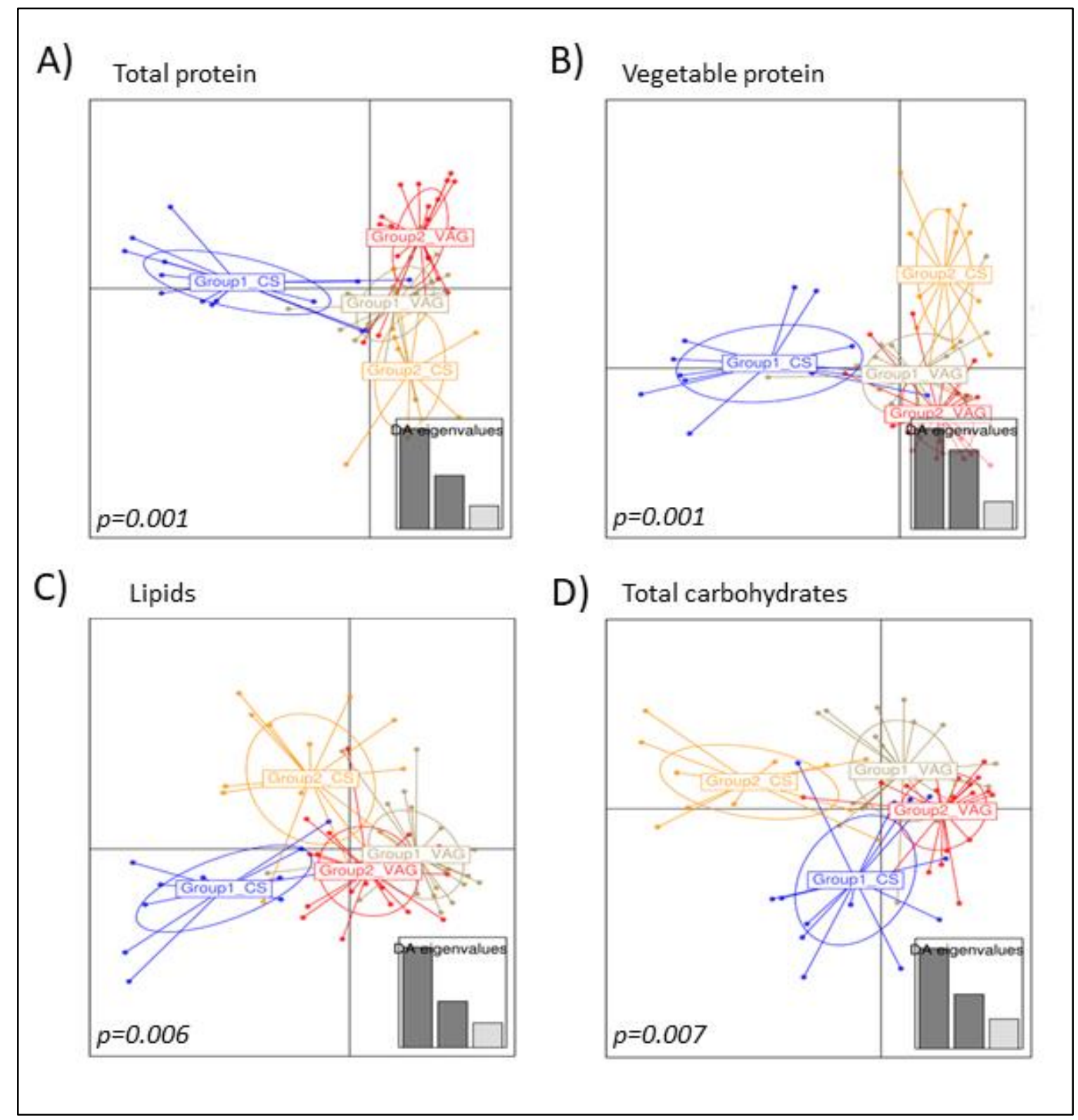

Supplementary file 6. Neonatal microbiota was distinctly shaped depending on mode of birth and maternal diet. Discriminant Analysis of Principal Components (DAPC) of the neonatal microbiota according to mode of delivery and maternal nutrients intake classification. VAG (Vaginal delivery), CS (C-Section), Group1/2: maternal consumption of the each nutrient lower (1)/higher (2) than average intake of the study population, including total protein (A) those from vegetal source (B), lipids (C) and total carbohydrates (D). 


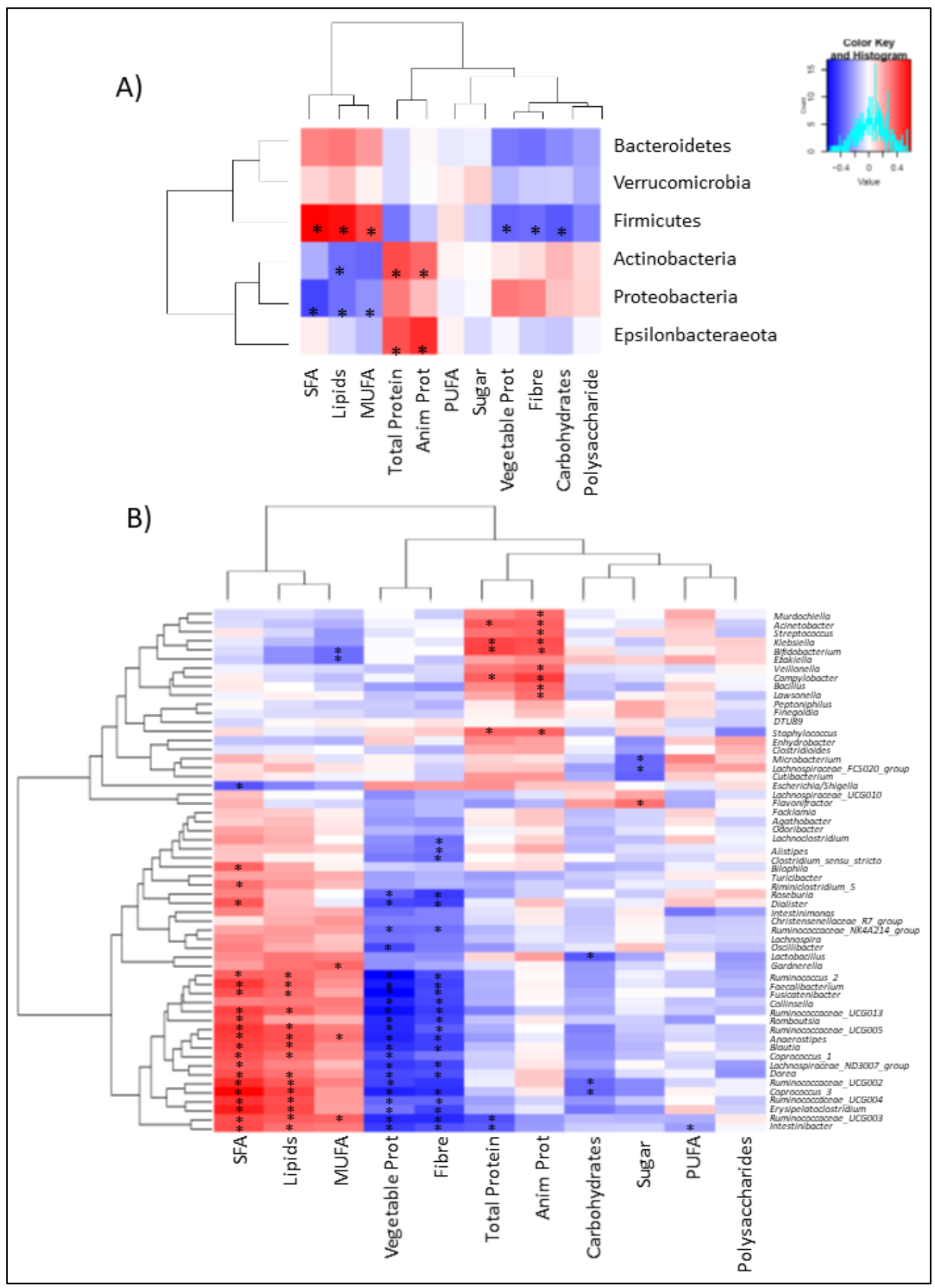

Supplementary file 7. Maternal diet was associated with neonatal microbiota at birth in vaginal born infants. Spearman correlations between maternal nutrients intake during pregnancy and neonatal bacterial composition at phylum (A) and genus level (B) at birth. Significant correlations $(p<0.05)$ were marked by an asterisk $(*)$. Red color represents the positive correlations whereas blue color shown negative relations. The most abundant genera were showed in the graph. Bacterial genera and dietary components are grouped at the square edges. Genera with some significant relation with maternal diet were shown. 


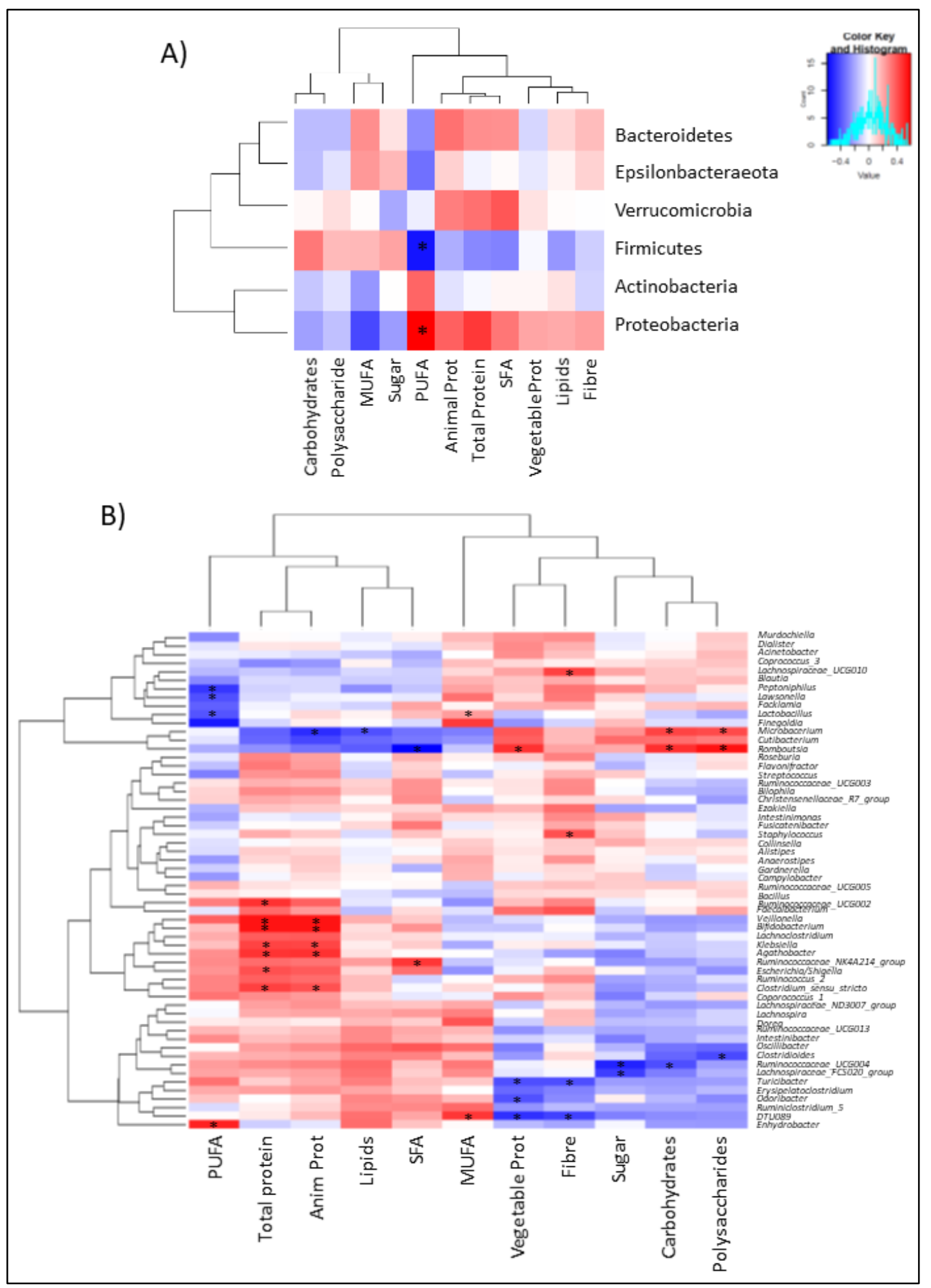

Supplementary file 8. Maternal diet was associated with neonatal microbiota at birth in Csection born infants. Spearman correlations between maternal nutrients intake during pregnancy and neonatal bacterial composition at phylum (A) and genus (B) level at birth. Significant correlations $(\mathrm{p}<.05)$ were marked by an asterisk $\left(^{*}\right)$. Red color represents the positive correlations whereas blue color shown negative relations. The most abundant genera were showed in the graph. Bacterial genera and dietary components are grouped at the square edges. Genera with some significant relation with maternal diet were shown. 